\title{
ESTIMULACIÓN SUPLEMENTARIA, MEMORIA A CORTO PLAZO Y ANSIEDAD EN JÓVENES
}

\author{
Ps. William Montgomery Urday
}

Se exploraron relaciones entre la afectividad y la cognición a través de un diseño factorial $2 \times 2$ con grupos pareados, asignándose 17 sujetos a cada grupo. El experimento consistió en administrar a sujetos con y sin ansiedad auto reportada, un tipo de estimulación suplementaria (una ilustración y dos textos) que evocara respuestas de corte emocional negativo (ira, miedo, tristeza o asco) luego de haber memorizado durante dos minutos una lista de palabras con connotaciones positivas, negativas y neutras. Las hipótesis principales giraron en torno a que los individuos con mayor o menor nivel de ansiedad tenderían, luego de haber sido expuestos a la estimulación suplementaria, a recordar más palabras de tipo emocional negativo que los de los demás grupos. Igualmente las personas ansiosas tenderían a lo mismo más que los otros sin necesidad de dicha estimulación. Esto fue confirmado plenamente sólo en cuanto al plano de la interacción entre las variables ansiedad estimulación suplementaria, concluyéndose que los estados de elevada activación ansiógena hacen a los sujetos más propensos a atender y recordar estímulos amenazantes o inquietantes.

PALABRAS CLAVE: Estimulación suplementaria, memoria a corto plazo, ansiedad, conducta verbal.

Is the purpose to explore some relations beetwen anxiety and cognition through a factorial design 2 X 2 with even groups of seventeen subjects each one. The experiment consist in to manage to subjects with autorefering anxiety and no that, a type of stimulation evoked negative emotional responses (angry, fear, sadness and sick), after to memorize during for two minutes a words list with positive, negative and neutral meaning. Its supposing the subjects with plus or minor anxiety level tends to memory more negative emotional words. This is confirmed only in the plane of simultaneous function anxiety-supplementary stimulation. Was concluded that high activation state anxiety maked prone the subjects pay any attention and remember threatening stimulus.

KEY WORDS: Supplementary stimulus, short place memory, anxiety, verbal behavior. 


\section{INTRODUCCIÓN}

A través del presente estudio se intentan explorar algunas relaciones entre la afectividad y la cognición, en el entendido de que la base de los procesos llamados superiores se halla, en gran parte, determinada por los hábitos verbales y retencionales aprendidos por el individuo, en conjunción con sus respuestas emocionales condicionadas (Miller, 1969, trad. esp.; Olerón, 1969, trad. esp.). Siendo patente la interacción entre los estados emotivos, la atención, la memoria y el discurso lingüístico, muchas investigaciones se han planteado, tanto a nivel educativo como clínico, el objetivo de esclarecer el papel de las emociones (en especial el interés, la ansiedad y la depresión) como un contexto para la adquisición y la recuperación de experiencias verbales, en el marco de la "memoria a corto plazo" u "operativa".

Los ramos principales de indagación en las aproximaciones empirista, gestáltica y psicoanalítica identifican esta memoria a nivel práctico, con los aprendizajes de tipo serial, de pares asociados y de libre recuerdo (véanse las reseñas de Lieury, 1978, trad. esp.; Aranda, 1990; y Baddeley, 1999, trad. esp.). Se indica que cuando una persona da una respuesta de discriminación verbal como "recuerdo" frente a un reactivo (o lista de reactivos) inmediatamente presentada antes, basaría su elección en: a) la frecuencia con que ha visto, oído y pronunciado la palabra anteriormente, b) las integraciones sintácticas que evocan operadores "apropiados" de acuerdo a leyes estructurales de la gramática, y c) la presencia de estados de ánimo específicos en el momento de responder, ligados a situaciones particulares en la historia personal.

Respecto al último punto poco se ha dicho, sin embargo, sobre las condiciones que "inducen" a responder en cierto tipo de tareas memorísticas cuando la fuerza de la activación emotiva (en especial la ansiedad) es más bien moderada, pese a existir como disposición. Cabe preguntarse si variables no explícitas (las cuales pueden denominarse "suplementarias") juegan el papel de catalizadores afectivo s en semejante contexto. Y si, además, la forma como lo hacen y sus características son de alguna manera interdependientes entre sí.

Comportamentalmente, pueden reformularse de manera técnica los puntos señalados, en el sentido de que los problemas respecto a una respuesta de "recuerdo" se referirían a conductas ecoica y textual llevadas al control intraverbal en el caso de la frecuencia con que se ha visto, oído y pronunciado la palabra anteriormente, a las respuestas autocríticas de relación en el caso de las integraciones sintácticas que evocan operadores "apropiados" de acuerdo a leyes estructurales de la gramática, y a las respuestas bajo control de condiciones especiales, como reflejos o disposiciones en el caso de la presencia de estados de ánimo específicos en el momento de responder, ligados a situaciones particulares en la historia personal (véanse Shimp, 1976; Branch, 1977; Skinner, 1981, trad. esp.; Palmer, 1999). En cuanto a las disposiciones, es de interés particular para este estudio la presunción de que en nuestro mundo social tales condiciones se hallan, para hacerse manifiestas, en 
contingencia con algún tipo de estimulación verbal discriminativa no directa ni evidente. En otras palabras, debe haber un contexto referencial que "dispone" a sujetos con fuerte o regular tendencia a experimentar activación emotiva, a "actualizar" o "aumentar" tal estado y que éste los incline a emitir ciertas operantes verbales conectadas a dicha activación, como lo muestra parcialmente un experimento clásico' de Kleinsmith y Kaplan en 1963 (cit. por Eysenck, 1985, trad. esp.; p. 286), al pedir a sus sujetos que se concentraran sobre una lista de ítems asociados por pares mientras se registraban sus reacciones cutáneas clasificadas como intensas o ligeras. En la tarea de recuerdo resultó que la activación y el intervalo de retención se relacionaban, siendo evidente que algunos ítems jugaban el papel de "disparadores" sobre ciertas respuestas activadoras.

Teniendo en cuenta que lo anterior se atribuye comúnmente al "significado" que tendrían los ítems en cuestión para cada individuo, es conveniente indicar que el origen del significado no es una propiedad de la respuesta o situación exclusivamente, sino de la contingencia en la cual también intervienen variables disposicionales (Corral, 1997), las cuales muchas veces son insuficientes para evocar por sí solas una respuesta y por ello requieren de una estimulación suplementaria para producirse. De acuerdo con Skinner (1981, trad. esp.), las variables suplementarias son condiciones que contribuyen a la emisión de una respuesta en una circunstancia en la cual, por distintos motivos, las fuentes de fortalecimiento de la tendencia a reaccionar son muy bajas (cabe referirse aquí, por ejemplo, a las operantes emitidas por un sujeto ante las tareas de asociación de palabras o de imágenes implícitas en las pruebas de Jung, de Rorschach o de Murray). Skinner (ibid, p. 57) cita una experiencia en el aula de clases, donde F. S. Keller expuso sucesivamente a sus estudiantes a dos textos casi idénticos escritos en la pizarra:

\section{5 \\ 4 \\ 4 \\ 3 \\ Hágalo en papel Hágalo en papel}

En el primer caso hablaba incidentalmente de sumas y adiciones, mientras que en el segundo incluía en su discurso muchas palabras que sugerían operaciones multiplicadoras. Por cierto que en ninguno de los dos momentos dijo lo que debía hacerse, pero al comparar el número de alumnos que multiplicaron con el del que sumaron, observó que eran muchos más los que habían multiplicado en el segundo caso. Aunque Skinner menciona este experimento para ilustrar ciertas propiedades dinámicas del mando, parece evidente que la variable en juego aquí es de tipo suplementario, ya que faltando el símbolo que definiría la emisión concreta de la conducta, la fuerza de ésta era débil, y sólo se produjo cuando del ambiente verbal circundante cada sujeto, en base a su propia historia de reforzamiento, "asimiló" la estimulación faltante.

En este sentido, probablemente lo que se ha llamado "inducción de estados de ánimo" para evaluar sus efectos sobre el recuerdo de palabras connotadas afectivamente con emociones de alegría o tristeza (Blasco, Borrás, Rey, Bonilla y Fernández, 1997), no es más que una forma de estimulación suplementaria en cualquiera de sus modalidades de presentación.

Son conocidos los efectos de fuertes variables disposicionales ansiógenas en los sujetos 
sobre su desempeño. Aparentemente, en quienes padecen de ansiedad latente, hay un "balance" de la preocupación y la distracción ocasionados por una tarea que involucra operaciones memorísticas, con el mayor esfuerzo que ellos suelen poner para superar el problema. Pero no siempre es así, en situaciones sencillas de aprendizaje verbal los sujetos con alto nivel de ansiedad aprenden más rápido, sucediendo lo contrario en tareas difíciles (Spence, 1958; cit. por Arnau, 1978; p. 107). Igualmente, las personas con ansiedad-rasgo parecen ser más conscientes de las palabras amenazantes, como lo muestran los experimentos de retención de palabras en función a su tinte afectivo (positivas, negativas o neutras) (Light \& CarterSobell, 1970; Eysenck, Mc. Leod \& Mathews, 1987; cits. por Baddeley, J 999, trad. esp.; p. 342; Blasco y cols., 1997), determinándose, como indica Flores (1975), grandes diferencias de capacidad de almacenamiento entre grupos de sujetos que tratan de retener información "agradable" y "desagradable" (véase también Ortiz Soria, 1999); y si se acepta, según la mayoría de tendencias teóricas actuales, que la auto estimulación verbal se vincula al equilibrio emotivo y funcional del individuo, hay que entender el riesgo que corre una persona con alta activación ansiógena respecto al tipo de lenguaje que usa para rotular los eventos del mundo, y para controlarse a sí mismo y a los demás, pudiendo derivar en situaciones de irritabilidad o depresión crónicas (Spielberger, 1980, trad. esp.), afectando también, por consiguiente, su estabilidad en el rendimiento lectivo.

Estas cuestiones son materia de la presente investigación, que puede ser de utilidad para aportar datos aplicables a problemas frecuentes en la educación superior, como los déficit de motivación, autocontrol, atención selectiva y capacidad de retención (Uriarte y Cabrera, 1981; Hernández Díaz, 1987), con el fin de arbitrar mejores estrategias para evitar fracasos y deserciones en la carrera de psicología y afines. Adicionalmente, podría generar conocimientos más elaborados en los planos de la significación verbal y la psicoterapia, en el curso de sucesivas investigaciones posteriores que complementen a ésta.

En base a lo dicho, se busca determinar: a) si una variable suplementaria influye significativa-mente sobre el número y tipo de palabras recordadas en una tarea de memorización a corto plazo, b) si la variable ansiedad influye significativamente sobre el número y tipo de palabras recordadas en una tarea de memorización a corto plazo, y c) si existe interacción significativa entre los efectos de las variables suplementaria y de ansiedad en la misma tarea.

En el proceso de verificación de estos objetivos se compararán los registros de palabras de connotación afectiva positiva, negativa y no afectiva entre los sujetos ansiosos y no ansiosos que reciben estimulación suplementaria y los sujetos ansiosos y no ansiosos que no reciben estimulación suplementaria, en sus diferentes combinaciones. 


\section{MARCO TEÓRICO}

El análisis experimental de la conducta es una forma teórica de tratar los problemas de la psicología, inspirada en la filosofía conductista. Desde dicha perspectiva se considera que dichos asuntos son abordables desde el plano de una ciencia del comportamiento (Skinner, 1979, trad. esp.; Kantor, 1991, trad. esp.), siendo éste el objeto de estudio que: a) ocurre dentro de un límite espaciotemporal cuya referencia necesita descubrirse en relación con un contexto geográfico, b) muestra un conjunto de aspectos manifiestos definibles por su ocurrencia objetiva, c) produce efectos en el medio físico, biológico y social, siendo a su vez afectada por aquel, y d) tiene propiedades dimensionables en términos paramétricos y probabilitarios (Ribes y López, 1985).

Las características anteriores gravitan en torno a una conceptualización del evento psicológico como un continuo interactivo en el cual la afectividad, la conducta motora y la cognición son aspectos de un mismo y único proceso organísmico relacionado con el ambiente físico biológico y social. Desde esta perspectiva se abordarán las definiciones de términos del tipo de conducta verbal, estimulación suplementaria, memoria como conducta de recordar y como repertorio, y ansiedad como disposición emocional condicionada.

1. Conducta verbal y estimulación suplementaria. De acuerdo con el enfoque de Skinner (1981, trad. esp.), la conducta verbal es aquella que se re fuerza a través de la mediación de otra persona, siendo una forma de comportamiento moldeado y mantenido por consecuencias que se producen a nivel social. Las principales categorías verbales son: a) el mando, bajo control funcional de condiciones 1 de privación o estimulación aversiva, b) el tacto, bajo control de eventos no verbales c) la conducta textual, bajo control de estimulación verbal no auditiva. d) la conducta intraverbal, cuya correspondencia con el estimulo verbal no tiene una equivalencia exacta, e) la conducta ecoica cuya correspondencia con el estímulo verbal si tiene una correspondencia exacta, y t) la conducta autocrítica, que designa aquella conducta verbal basada en otra, o que depende de ella.

La estimulación suplementaria es una condición que contribuye a que una respuesta pueda ser emitida, dentro de una situación en que por determinadas razones las fuentes de fortalecimiento existente son insuficientes para que la conducta se emita. Las variables suplementarias pueden ser de instigación (cuando quien pone la tarea conoce la respuesta que pretende evocar en el sujeto), o sondeo (cuando el experimentador y el sujeto desconocen la respuesta que podría aparecer).

2. Memoria. Dentro de la perspectiva reseñada se concibe: a) como conducta de recordar, y b) como repertorio. Debe notarse que en casi todos los casos un sujeto inmerso en una situación "memorística" maneja determinadas variables (auto sondeos) para producir una respuesta que no se puede identificar de antemano (Skinner, 1979, trad. esp.). Por ejemplo, cuando se dice a sí mismo "la he visto en alguna parte... ", fortalece la posibilidad de acordarse de una cara. Esta posibilidad de responder existe, además, como una entre un conjunto de conductas latentes que el sujeto es capaz de producir en cierto momento, con base en adquisiciones anteriores (Catania, 1974, trad. esp.).

En el plano de la evocación inmediata, Baddeley y Hitch (cit. por Eysenck, 1985, trad. esp., p. 60) introdujeron una distinción entre los términos de "memoria a corto plazo" y 
"memoria operativa", indicando que la segunda denominación designa mucho más completamente sus propiedades, al conceptuarla, además de como un mecanismo de procesamiento atencional, acompañado de un bucle' articulatorio que permite almacenar provisionalmente una cantidad limitada de información en un código fonémico.

3. Ansiedad. Se concibe como un estado emocional condicionado (en relación con el sistema nervioso autónomo) que surge como respuesta a estímulos anteriormente vinculados a situaciones perturbadoras. Su patrón de ocurrencia se configura disposicionalmente en el organismo, aumentando la probabilidad de reacciones de regular o fuerte activación según las condiciones ambientales. Spielberger (1980, trad. esp.) indica que dicha activación puede manifestarse como rasgo (si es una disposición orgánica o arraigada muy tempranamente), o como estado (si constituye una reacción a circunstancias de momentánea o crónica problematicidad. En el estudio clásico de Estes y Skinner (1974, trad. esp.) se fijaron las dos principales características que definen la ansiedad: a) tiene una gran semejanza con el temor, y b) obedece a una "anticipación" por parte del organismo de la ocurrencia futura del estimulo perturbador.

\section{MÉTODO}

\section{Diseño}

Se utilizo un diseño experimental de tipo factorial $2 \times 2$ con grupos pareados, considerando las variables en el orden siguiente:

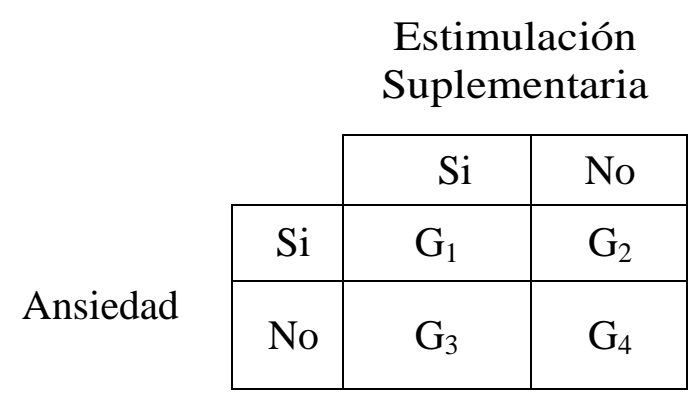

\section{Sujetos}

Se consideró como población total un conjunto de 250 alumnos del curso de Métodos del Trabajo Universitario durante dos semestres, todos estudiantes de primer año de psicología en la UNMSM, de ambos sexos y con edades entre 17 a 21 años. La selección de los participantes se hizo en base al método de grupos pareados, utilizando como variables de pareamiento: a) el número total de palabras recordadas, y b) el puntaje medio y alto alcanzado en la subescala de Asimilación de Conocimientos perteneciente al Inventario de Hábitos de Estudio de J. Pozar. De allí se obtuvo una muestra total compuesta por 68 individuos, asignándose cuatro grupos de 17 sujetos cada uno. 


\section{Variables}

\section{1) Independientes}

a) Estimulación suplementaria, consistente en la presentación de dos textos en forma de frases incompletas: "Cuando veo el mundo, creo... ", "Cuando veo mi vida, pienso ... ", en la parte inferior de una página que muestra en la parte superior cuatro rostros humanos expresando estados emocionales de ira, miedo, tristeza y asco.

b) Nivel de ansiedad auto reportado en el puntaje de la prueba de ansiedad de Zung.

\section{2) Dependiente}

Número de palabras recordada en sentido de connotación afectiva positiva, negativa y neutra, elegidas por método de Jueces:

Palabras de connotación positiva.

Héroe, Fiesta, Belleza, Elogio, Canción, Amor, Gracia, amistad, Valor, Paseo, Premio, Calma, Flor, Placer, Lujo Dinero, Alegría.

Palabras de connotación negativa.

Odio, Desdén, Miedo, Soledad, Crueldad. Víctima, Traición, Duda, Cólera, Llanto, Temor, Perdón, Castigo, Crisis, Pena, Atraso, Condena.

Palabras de connotación neutra Papel, Calle, Lápiz, Vela, Era, Sala, Llave, Medio, Piso, Marco, Suela, Torre, Balde, Pared, Timón, Orilla.

\section{3) De control}

Edad, Grado Académico, puntaje en la escala de asimilación de conocimientos.

\section{Instrumentos}

1. Escala de Autovaloración de la Ansiedad de Zung., conteniendo una escala de veinte afirmaciones acerca de estados fisiológicos y emocionales altamente activados, con calificación subjetiva tipo Likert 1 - 4, 4 -1. Un puntaje bajo (menos de 45) no connota ansiedad, mientras que de 45 hasta 74 la indica en diversos grados. Para el presente estudio su nivel de confiabilidad fue establecido mediante la Fórmula Profética de Spearman-Brown, obteniendo un índice de 0.93. La validez del contenido se determinó mediante la correlación item test corregida.

2. Sub-Escala de Asimilación de Conocimientos perteneciente al Inventario de Hábitos de Estudio de J. Pozar, conformada por quince ítems de calificación triádica SI - ? No. Únicamente se utilizó como variable de apareamiento. Los datos globales, ya convenientemente confiabilizados, validados y baremados fueron obtenidos del archivo de la Oficina de Asesoramiento y Orientación al Estudiante (OFAYOE) de la universidad.

\section{Procedimientos}

1. Aplicación de la Prueba de Ansiedad de Zung y división de la muestra en dos grupos generales: "Ansiosos moderados" (de 45 a 49 puntos) y "No Ansiosos" (de 44 a 
menos).

2. Especificación de cuatro grupos de 17 sujetos cada cual, de acuerdo con la siguiente clasificación:

a) Sin ansiedad, que recibe estimulación suplementaria.

b) Sin ansiedad, que no recibe estimulación suplementaria.

c) Con ansiedad, que recibe estimulación suplementaria.

d) Con ansiedad, que no recibe estimulación suplementaria.

3. Administración de un formato conteniendo 50 palabras de connotación afectiva positiva, negativa y neutra intercaladas, con la indicación a los sujetos de que tienen dos minutos para memorizar todas las que puedan.

4. Administración de la variable independiente de estimulación suplementaria.

5. Administración de un formato preparado para ser llenado con todas las palabras recordadas, indicando a los sujetos un tiempo máximo de dos minutos para hacerlo.

Se hizo el análisis estadístico en base a las pruebas de Análisis de Varianza de 2 factores Total, Intra y Entre grupos, para determinar la influencia de las variables independientes y su interrelación, así como la Prueba $F$ para determinar las diferencias que pudieran haber ocurrido por azar. Adicionalmente, Se aplicó la Prueba $T$ para las comparaciones entre los índices alcanzados por cada uno de los grupos.

\section{RESULTADOS}

De la constatación empírica se obtienen los siguientes resultados, ilustrados en las tablas que figuran a continuación. En la tabla 4.1 (primera y segunda partes) se muestra la totalidad de las palabras recordadas por cada uno de los sujetos en cada grupo formado y categoría de recordación, así como los puntajes de las variables de pareamiento (asimilación de contenidos y número total de palabras recordadas) para la conformación de los grupos.

En la fig. 4.1 Se recogen los promedios alcanzados por cada uno de los grupos, observándose una clara predominancia del de sujetos ansiosos que fueron expuestos a la variable de estimulación suplementaria, en lo que respecta a las palabras de connotación afectiva negativa. Otra diferencia notoria es la vista en la comparación entre sujetos ansiosos con estimulación suplementaria y ansiosos no expuestos a ella en cuanto al recuerdo de palabras neutras, con ventaja para los últimos.

A través del Análisis de Varianza se constata el orden en que difieren los grupos entre sí. La mayor homogeneidad Se encuentra en la proporción de palabras afectivas positivas, luego la de palabras afectivas negativas y finalmente la de neutras (Tabla 4.2).

El análisis se completa en las tablas 4.3, 4.4. y 4.5, donde la Prueba $F$ confirma que el nivel de probabilidad es mayor que 0.05 en los casos de la interacción entre variables independientes respecto a las palabras de connotación afectiva negativa (Tabla 4.4), y de la variable ansiedad respecto a las palabras de connotación no afectiva o neutra (Tabla 4.5), rechazándose las respectivas hipótesis de nulidad, dado que el valor necesario para fijar la confiabilidad es de 4.00. En todos los demás casos Se acepta la hipótesis de nulidad. 
Finalmente, la Tabla 4.6 integra los resultados de las comparaciones entre los diferentes grupos mediante la Prueba T. Allí se observan diferencias significativas entre sujetos Ansiosos con Estimulación Suplementaria y Ansiosos sin ella, respecto a la proporción de palabras afectivas negativas y muy significativas respecto a las palabras no afectivas o neutras. Igualmente, los sujetos Ansiosos y No Ansiosos, ambos con Estimulación Suplementaria, muestran diferencias significativas en el índice de palabras afectivas negativas. También los Ansiosos con Estimulación Suplementaria se diferencian de los no ansiosos sin ella en cuanto a las palabras neutras, y de la confrontación de los No ansiosos con Estimulación Suplementaria versus los No Ansiosos sin ella, se siguen diferencias notables tanto en palabras afectivas positivas como en negativas.

TABL.A 4.1 (Primera parte)

PUNTAJES DE SUJETOS "ANSIOSOS" Y "NO ANSIOSOS" EN ASIMIL.ACIÓN DE.

CONOCIMIENTOS (AC), NÚMERO TOTAL DE PALABRAS RECORDADAS (T), Y NÚMERO DE PALABRAS AFECTIVAS POSITIVAS (E+), NEGATIVAS (E-) Y NEUTRAS (E N) RECORDADAS

\begin{tabular}{cccccccccccc}
\hline Ans. & $\mathbf{A C}$ & Total & $\mathbf{E}+$ & $\mathbf{E}-$ & $\mathbf{E}$ N & No Ans. & $\mathbf{A C}$ & Total & $\mathbf{E}+$ & $\mathbf{E}-$ & $\mathbf{E}$ N \\
\hline$\Sigma$ & 116 & 142 & 46 & 46 & 51 & $\sum^{2}$ & 116 & 142 & 41 & 54 & 47 \\
\hline$\Sigma^{2}$ & & & 144 & 144 & 170 & $\Sigma^{2}$ & & & 123 & 142 & 155 \\
\hline $\mathrm{X}$ & 6.82 & 8.35 & 2.70 & 2.70 & 3.00 & $\mathrm{X}$ & 6.82 & 8.35 & 2.41 & 3.18 & 2.76 \\
\hline
\end{tabular}

TABLA 4. 1 (Segunda parte)

PUNTAJES DE SUJETOS "ANSIOSOS CON ESTIMULACIÓN SUPLEMENTARIA" Y "NO ANSIOSOS CON ESTIMULACIÓN SUPLEMENTARIA" EN ASIMILACIÓN DE CONOCIMIENTOS (AC), NÚMERO TOTAL DE PALABRAS RECORDADAS (T), Y NÚMERO DE PALABRAS AFECTIVAS POSITIVAS (E+), NEGATIVAS (E) Y NEUTRAS (EN) RECORDADAS

\begin{tabular}{cccccccccccc}
\hline Ans. c/Es & $\mathbf{A C}$ & Total & $\mathbf{E}+$ & $\mathbf{E}-$ & $\mathbf{E N}$ & No Ans, c/Es & $\mathbf{A C}$ & Total & $\mathbf{E}+$ & $\mathbf{E}-$ & $\mathbf{E}$ \\
\hline$\Sigma$ & 116 & 142 & 50 & 62 & 30 & $\sum_{2}$ & 116 & 142 & 51 & 46 & 45 \\
\hline$\Sigma^{2}$ & & & 172 & 254 & 72 & $\Sigma^{2}$ & & & 173 & 146 & 157 \\
\hline $\mathrm{X}$ & 6.82 & 8.35 & 2.94 & 3.65 & 1.76 & $\mathrm{X}$ & 6.82 & 8.35 & 3.00 & 2.71 & 2.65 \\
\hline
\end{tabular}

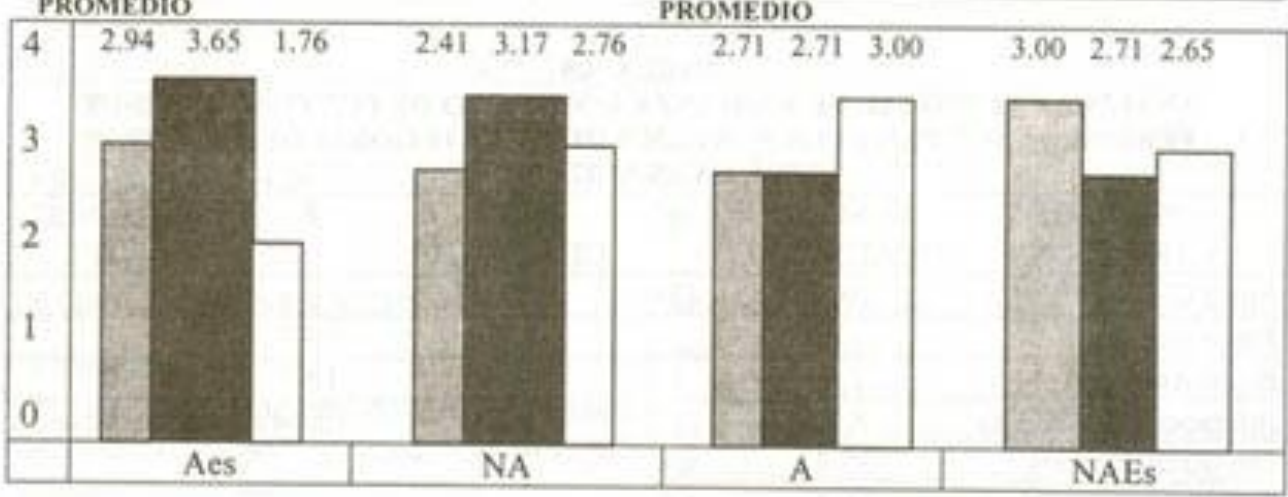

Fig. 4.1. Promedios de cada grupo de comparación.

Aes : Ansiosos con aplicación de estimulación suplementaria.

NA : No ansiosos.

A : Ansiosos.

NAes: No Ansiosos con aplicación de estimulación suplementaria. 
Tabla 4.2

ANÁLISIS DE VARIANZA POR CATEGORIAS DE PALABRAS RECORDADAS

\begin{tabular}{|lccc|}
\hline CATEGORIAS & SS INTRA GRUPOS & SS ENTRE GRUPOS & SS TOTAL \\
\hline $\begin{array}{l}\text { Palabras afectivas } \\
\text { Positivas }\end{array}$ & 88.59 & 3.65 & 92.24 \\
\hline $\begin{array}{l}\text { Palabras afectivas } \\
\text { Negativas }\end{array}$ & 35.41 & 10.50 & 45.91 \\
\hline $\begin{array}{l}\text { Palabras no afectivas } \\
\text { Neutras }\end{array}$ & $99 . .00$ & 14.87 & 113.87 \\
\hline
\end{tabular}

Tabla 4.3

ANÁLISIS FACTORIAL DE VARINZA COMPLETO DE PUNTUACIONES DE PERFORMANCE PARA LOS PUNTAJES DE LA CATEGORÍA DE PALABRAS AFECTIVAS POSITIVAS

\begin{tabular}{|lccccc|}
\hline $\begin{array}{l}\text { FUENTE DE } \\
\text { VARIACIÓN }\end{array}$ & $\begin{array}{c}\text { SUMA DE } \\
\text { CUADRADAS }\end{array}$ & $\boldsymbol{d} \boldsymbol{c}$ & $\begin{array}{c}\text { MEDIA } \\
\text { CUADRADA }\end{array}$ & F & $\begin{array}{c}\text { VALOR NEC.: } \\
\mathbf{4 . 0 0}\end{array}$ \\
\hline Entre Grupos & 3.65 & $(3)$ & & & \\
\hline Entre Es. Sup. & 0.24 & 1 & 0.23 & 0.17 & - \\
\hline Entre Ansiedad & 2.88 & 1 & 2.88 & 2.08 & - \\
\hline $\begin{array}{l}\text { Interacción Es X } \\
\text { Ans }\end{array}$ & 0.53 & 1 & 0.53 & 0.38 & - \\
\hline Dentro de Grupos & 88.59 & 64 & 1.38 & & \\
\hline Total & 95.88 & 67 & & & \\
\hline
\end{tabular}

Tabla 4.4

ANÁLISIS FACTORIAL DE VARIANZA COMPLETO DE PUNTUACIONES DE PERFOMANCE PARA LOS PUNTAJES DE LA CATEGORÍA DE PALABRAS AFECTIVAS NEGATIVAS

\begin{tabular}{|lccccc|}
\hline $\begin{array}{l}\text { FUENTE DE } \\
\text { VARIACIÓN }\end{array}$ & $\begin{array}{c}\text { SUMA DE } \\
\text { CUADRADAS }\end{array}$ & $\boldsymbol{d} f$ & $\begin{array}{c}\text { MEDIA } \\
\text { CUADRADA }\end{array}$ & F & $\begin{array}{c}\text { VALOR NEC.: } \\
\mathbf{4 . 0 0}\end{array}$ \\
\hline Entre Grupos & 10.50 & $(3)$ & & 1.97 & - \\
\hline Entre Es. Sup. & 1.09 & 1 & 1.09 & 1.97 & - \\
\hline Entre Ansiedad & 1.09 & 1 & 1.09 & 15.04 & - \\
\hline $\begin{array}{l}\text { Interacción Es X } \\
\text { Ans }\end{array}$ & 8.32 & 1 & 8.32 & & \\
\hline Dentro de Grupos & 35.41 & 64 & 0.55 & & \\
\hline Total & 56.41 & 67 & & & \\
\hline
\end{tabular}


TABLA 4.5

ANÁLISIS DE VARIANZA COMPLETO DE PUNTUACIONES DE PERFOMANCE PARA LOS PUNTAJES DE PALABRAS NO AFECTIVAS (NEUTRAS)

\begin{tabular}{|lccccc|}
\hline $\begin{array}{l}\text { FUENTE DE } \\
\text { VARIACIÓN }\end{array}$ & $\begin{array}{c}\text { SUMA DE } \\
\text { CUADRADAS }\end{array}$ & $\boldsymbol{d f}$ & $\begin{array}{c}\text { MEDIA } \\
\text { CUADRADA }\end{array}$ & F & $\begin{array}{c}\text { VALOR NEC.: } \\
\mathbf{4 . 0 0}\end{array}$ \\
\hline Entre Grupos & 14.87 & $(3)$ & & & \\
\hline Entre Es. Sup. & 1.78 & 1 & 1.78 & 1.15 & - \\
\hline Entre Ansiedad & 7.78 & 1 & 7.78 & 5.03 & Confiable \\
\hline $\begin{array}{l}\text { Interacción Es X } \\
\text { Ans }\end{array}$ & 5.31 & 1 & 5.31 & 5.43 & - \\
\hline Dentro de Grupos & 99.00 & 64 & 1.55 & & \\
\hline Total & 126.74 & 67 & & & \\
\hline
\end{tabular}

Tabla 4.6

ANÁLISIS COMPARATIVO DE PRUBAS T PARA LA INTERACCIÓN DE TODAS LAS CONDICIONES DE SUJETOS EN TODAS LAS CATEGORIAS DE PALABRAS

\begin{tabular}{|llll|}
\hline $\begin{array}{l}\text { Sujetos/ } \\
\text { Categorias }\end{array}$ & $\begin{array}{l}\text { palabras } \\
\text { afectivas } \\
\text { positivas }\end{array}$ & $\begin{array}{l}\text { palabras } \\
\text { afectivas } \\
\text { negativas }\end{array}$ & $\begin{array}{l}\text { palabras } \\
\text { no afectivas } \\
\text { (neutras) }\end{array}$ \\
\hline $\begin{array}{l}\text { Ansiosos c/Es vs } \\
\text { Ansiosos }\end{array}$ & 1.08 & $3.07^{*}$ & $4.15^{*}$ \\
\hline $\begin{array}{l}\text { Ansiosos c/Es vs } \\
\text { No ansiosos c/Es }\end{array}$ & 0.25 & $3.20^{*}$ & 2.07 \\
\hline $\begin{array}{l}\text { Ansiosos c/Es Sup vs } \\
\text { No ansiosos }\end{array}$ & 2.07 & $1.76^{*}$ & $3.11^{*}$ \\
\hline $\begin{array}{l}\text { Ansiosos vs } \\
\text { No ansiosos c/Es }\end{array}$ & 1.31 & 0.00 & 1.06 \\
\hline $\begin{array}{l}\text { No ansiosos c/Es vs } \\
\text { No ansiosos }\end{array}$ & 2.68 & 2.40 & 0.67 \\
\hline $\begin{array}{l}\text { Ansiosos vs } \\
\text { No ansiosos }\end{array}$ & 1.15 & 1.76 & 1.22 \\
\hline
\end{tabular}

* Significativo al 0.05 


\section{CONCLUSIONES Y DISCUSIÓN}

\section{Generales}

1. La presentación de una variable suplementaria, por sí sola, no influyó confiablemente sobre el número y tipo de palabras recordadas en la tarea de memorización a corto plazo.

2. La variable ansiedad no influyó confiablemente sobre el número de palabras afectivas recordadas en la tarea de memorización a corto plazo, pero si sobre las neutras.

3. Existió interacción con fiable entre los efectos de las variables suplementarias y de ansiedad en lo que respecta al rubro de palabras afectivas de connotación negativa recordadas en la tarea de memorización a corto plazo.

\section{Específicas}

1. Los sujetos ansiosos que recibieron estimulación suplementaria recordaron significativamente más palabras de connotación afectiva negativa que los sujetos ansiosos que no reciben estimulación suplementaria.

2. Los sujetos ansiosos que recibieron estimulación suplementaria recordaron significativamente más palabras de connotación afectiva negativa que los sujetos no ansiosos que reciben estimulación suplementaria.

3. No hubo diferencias significativas entre los sujetos ansiosos que recibieron estimulación suplementaria y los sujetos no ansiosos que no recibieron estimulación suplementaria. en cuanto al recuerdo de palabras de connotación afectiva negativa.

4. No hubo diferencias significativas entre los sujetos ansiosos que no recibieron estimulación suplementaria y los sujetos no ansiosos que recibieron estimulación suplementaria, en cuanto al recuerdo de palabras de connotación afectiva negativa.

5. No hubo diferencias significativas entre los sujetos no ansiosos que recibieron estimulación suplementaria y los sujetos no ansiosos que no recibieron estimulación suplementaria, en cuanto al recuerdo de palabras de connotación afectiva negativa.

6. No hubo diferencias significativas entre los sujetos ansiosos que no recibieron estimulación suplementaria y los sujetos no ansiosos que no recibieron estimulación suplementaria, en cuanto al recuerdo de palabras de connotación afectiva negativa.

7. No hubo diferencias significativas entre los sujetos ansiosos que recibieron estimulación suplementaria y los sujetos ansiosos que no recibieron estimulación suplementaria, en cuanto al recuerdo de palabras de tipo afectivo positivo y/o neutro. 
8. No hubo diferencias significativas entre los sujetos ansiosos que recibieron estimulación suplementaria y los sujetos no ansiosos que recibieron estimulación suplementaria, en cuanto al recuerdo de palabras de tipo afectivo positivo y/o neutro.

9. Se halló diferencias significativas entre los sujetos ansiosos que recibieron estimulación suplementaria y los sujetos no ansiosos que no recibieron estimulación suplementaria, en cuanto al recuerdo de palabras de tipo neutro.

10. No hubo diferencias significativas entre los sujetos ansiosos que no recibieron estimulación suplementaria y los sujetos no ansiosos que recibieron estimulación suplementaria. en cuanto al recuerdo de palabras de tipo afectivo positivo y/o neutro.

11. Se halló diferencias significativas entre los sujetos no ansiosos que recibieron estimulación suplementaria y los sujetos no ansiosos que no recibieron estimulación suplementaria, en cuanto al recuerdo de palabras de tipo afectivo positivo $\mathrm{y}$ negativa, con ventaja para los no ansiosos.

12. No hubo diferencias significativas entre los sujetos ansiosos que no recibieron estimulación suplementaria y los sujetos no ansiosos que no recibieron estimulación suplementaria, en cuanto al recuerdo de palabras de tipo afectivo positivo y/o neutro.

Los sujetos calificados como "ansiosos" de acuerdo con los estándares de la prueba de Zung, sometidos a una modalidad de estimulación suplementaria como la presentación de imágenes y textos evocadores de respuestas condicionadas desagradables, tendieron significativamente a recordar palabras de connotación emotiva en consonancia con inquietudes de pesimismo y preocupación, lo que concuerda con los resultados de Blasco y cols. (1997), ya citados. En este sentido la conjetura principal del estudio, la interacción significativa entre las variables ansiedad y estimulación suplementaria, fue confirmada, mostrando que ésta última tiene una alta probabilidad de inducir conductas en personas con un estado (o rasgo) emocional específico, aun cuando dicha condición sea de intensidad moderada.

Así como el aumento del grado de motivación es correlativo al aumento de la eficiencia y la memorización (Flores, 1975; Lieury, 1978, trad. esp.), y así como la depresión puede producir una ralentización de las 'respuestas o hacerlas tender a recuerdos tristes (Baddeley, 1999, trad. esp.), no sorprende que el nivel elevado de ansiedad produzca tendencias a percibir con mayor atención estímulos considerados amenazantes o desagradables.

Según Craik y Lochart (cit. por Aranda, 1990, p. 59), los repertorios memorísticos se ligan con la percepción y el reconocimiento de patrones. Entendida como un "plan de recuperación de recuerdos", la memoria a corto y a largo plazo se vale, en primer lugar, de la exclusión de ciertos datos "irrelevantes" (es decir, probablemente ni reforzantes ni 
aversivos para el organismo), y, en segundo lugar, de una "reelaboración" de la información añadiéndole datos congruentes con los moldes asociativos habituales que tiene cada individuo. Si a ese proceso natural se le adjunta estimulación suplementaria, la fuerza de la respuesta resulta lógicamente incrementada.

Un resultado que coadyuva a la confirmación de la hipótesis es el obtenido a través de la comparación entre sujetos ansiosos que fueron sometidos al influjo de la variable suplementaria y sujetos no ansiosos que no la experimentaron. Allí se puede observar una diferencia significativa en la evocación de palabras neutras a favor de los últimos. Dado que ambos grupos representan respectivamente los dos extremos de presencia y ausencia de variables independientes, era esperable que el conjunto de sujetos con presencia de tales variables mostraran mucho menor volumen de recordación no afectiva.

En la misma línea quizá debiera haberse verificado que en la comparación entre los sujetos no ansiosos, aquellos sometidos a la estimulación suplementaria tuvieran mayor incidencia de evocación de palabras negativas que positivas, pero curiosamente sucedió todo lo contrario. Los sujetos no estimulados suplementariamente obtuvieron resultados significativos sobre el grupo opuesto al recordar bastante menos palabras de connotación positiva y bastante más palabras de connotación negativa. Eso podría explicarse, con cierta probabilidad, sobre la base del razonamiento siguiente: los individuos que carecen de ansiedad tenderían a exhibir respuestas de afrontamiento ante la aparición de estímulos "amenazante s" para su salud mental. Así, al someterlos a la estimulación suplementaria seleccionada para producir reacciones negativas, la mayoría habría respondido imponiendo un "filtro" de atención que descarte aquellas, centrándose más bien en lo positivo.

En suma, la estimulación suplementaria sólo funcionaría en la dirección deseada cuando actúa sobre sujetos moderada o altamente susceptibles a emociones evocadoras de ansiedad, lo que sugeriría la necesidad de procedimientos de manejo diferencial con respecto a variables contextuales o subliminales que, entre otras cosas, rodeen a personas (por ejemplo estudiantes) con niveles de estados de ánimo habitualmente inclinados al autocontrol o a la falta de éste.

Los resultados reseñados deben, desde luego, ser verificados a través de un estudio mayor. El uso de un diseño experimental de grupos pareados limita, hasta cierto punto, la obtención de una muestra grande, de allí el corto volumen de sujetos asignados a cada grupo (17) y la imposibilidad de comparar los resultados por sexo, variable que no se pudo parear debido a las desproporciones encontradas en este plano.

\section{REFERENCIAS}

Aranda, E. (1990). Anamnesis: Psicología de la memoria y el olvido. México: Trillas.

Amau, J. (1979). Motivación y conducta. Barcelona: Fontanella.

Baddeley, A. (1999, trad. esp.). Memoria humana: Teoría y práctica. Madrid: McGrawHill Interamericana.

Blasco, T.; Borrás, F.X.; Rey, M.; Bonilla, A. y Femández, J. (1997). Efectos de un procedimiento de inducción de estados de ánimo sobre el recuerdo de palabras. Anales de Psicología, 13(2), 163-176. 
Branch, M.N. (1977). On the role of "memory" in the analysis. of behavior. Journal of Experimental Analysis of Behavior, 26, 113-130.

Corral, V. (1997). Disposiciones psicológicas: Un análisis de las propensiones, capacidades y tendencias del comportamiento. México: UNISON.

Estes, W. K. y Skinner, B. F. (1974, trad. esp.). Algunas propiedades cuantitativas de la ansiedad (pp. 320-327). En Ch. Catania (Ed.). Investigaciones contemporáneas en conducta operante.

Eysenck, H. J. (1985, trad. esp.). Atención y activación. Barcelona: Herder.

Flores, C. (1975). La memoria. Vilasar de Mar: Oikos Tau.

Hemández-Díaz, F. (1988). Métodos y técnicas de estudio en la universidad. México: McGraw-Hill Latinoamericana.

Kantor, J. R. (1991, trad. esp.). La evolución científica de la psicología. México: Trillas.

Lieury, A. (1978, trad. esp.). La memoria: Resultados y teorías. Barcelona: Herder.

López Soria, B. (1999). El constructo de intensidad afectiva: Una revisión. Revista Electrónica de Motivación y Emoción.

Reme.uji.es/artículos/aotib 16826199/texto.2(2-3).

Miller, G. A. (1969, trad. esp.). Lenguaje y comunicación. Buenos Aires: Amorrortú.

Olerón, P. (1969, trad. esp.). Los hábitos verbales. En J. Piaget, P. Fraisse y otros (Eds.). Introducción a la psicolinguística. Buenos Aires: Proteo.

Palmer, D. C. (1999). A behavioral interpretation of memory. En LJ. Hayes y P.N. Chase (Eds.). Dialogues on verbal behavior. Reno: Context Press.

Pozar, J. (1981, trad. esp.). Inventario de hábitos de estudio. Madrid: TEA.

Ribes, E. y López, F. (1985). Teoría de la conducta: Un análisis de campo y paramétrico. México: Trillas.

Salas, E. (1987). La programación de la evaluación académica y su relación con la memoria a largo plazo en estudiantes del quinto ciclo de psicología de una universidad particular. UPCH. Tesis de Licenciatura, Lima.

Shimp, L. P. (1976). Organization in memory and behavior. Journal of the Experimental Analysis of Behavior, 28,171-179.

Skinner, B. F. (1979, trad. esp.). Ciencia y conducta humana. Barcelona: Fontanella.

Skinner, B. F. (1981, trad. esp.). Conducta verbal. México: Trillas.

Spielberger, Ch. (1980). Tensión y ansiedad. México: Harla.

Zung, W. K. (s/f). Test de Zung. Escala de Autoevaluación de la Ansiedad (EAA). Lima: S/e. 